for example an induction motor. The magnetic force producing the torque moves with the synchronous angular velocity of the alternating current, whereas the rotor speed falls below this by the slip, so that the magnetic force is moving relative to the rotor on which it is producing a torque. The rate of work done on the rotor is the torque multiplied by the rotor speed, and not the torque multiplied by the synchronous speed. In many cases, as in this case, the difference in these two quantities may be lost as mechanical work; in this case it appears as eddy current loss. The energy balance at the point of application is a function of the detailed method of application of the force. A problem, in which a similar difficulty in the use of partial and convected derivatives arose, appeared in the discussion of the motion of a bar containing a hinge moving along its length. ${ }^{2}$ In this case the wrong choice involved an apparent paradox: the failure of the momentum principle.

\title{
NOTE ON THE LEAST EIGENVALUE OF THE HILL EQUATION
}

By TOSIO KATO (University of Tokyo)

Let us consider the Hill equation

$$
y^{\prime \prime}+[\lambda+f(x)] y=0, \quad-\infty<x<\infty,
$$

where $f(x)$ is a real-valued periodic function of period 1 with the Fourier series

$$
f(x) \sim \sum_{n=-\infty}^{\infty} c_{n} \exp (2 \pi i n x), \quad \bar{c}_{n}=c_{-n} .
$$

Recently Wintner([5], Eq. (23)) deduced the following inequalities satisfied by the lower limit $\lambda_{0}$ of the spectrum of (1):

$$
-c_{0} \geqq \lambda_{0} \geqq-c_{0}-2 \sum_{n=1}^{\infty}\left|c_{n}\right|^{2} .
$$

Also the question is raised (Putnam [3], p. 314) whether the coefficient 2 on the righthand side is the least possible value. In the present note we shall show that better estimates do exist. In particular, we shall show that

$$
\lambda_{0} \geqq-c_{0}-\frac{1}{8} \sum_{n=1}^{\infty}\left|c_{n}\right|^{2} .
$$

For this purpose we note that $\lambda_{0}$ is characterized as the least eigenvalue of (1) considered on the finite interval $0 \leqq x \leqq 1$ with the periodic boundary conditions

$$
y(0)=y(1), \quad y^{\prime}(0)=y^{\prime}(1)
$$

(see e.g. Strutt [4], p. 15). Therefore, according to the Ritz variational principle, $\lambda_{0}$ is the minimum value of the expression

$$
J[y]=\int_{0}^{1}\left(y^{\prime 2}-f y^{2}\right) d x / \int_{0}^{1} y^{2} d x,
$$


where $y$ changes over real-valued functions subjected to the conditions (4). Let $z(x)$ be any periodic function of period 1 with integrable $z^{\prime}(x)$. Then we have

$$
\int_{0}^{1}\left(2 z y y^{\prime}+z^{\prime} y^{2}\right) d x=\left[z y^{2}\right]_{0}^{1}=0
$$

since both $y$ and $z$ are periodic. Hence

$$
\begin{aligned}
\int_{0}^{1}\left(y^{\prime 2}-f y^{2}\right) d x=\int_{0}^{1}\left[\left(y^{\prime}+z y\right)^{2}+\left(z^{\prime}-z^{2}-f\right) y^{2}\right] d x & \\
& \geqq \operatorname{Min}\left(z^{\prime}-z^{2}-f\right) \cdot \int_{0}^{1} y^{2} d x .
\end{aligned}
$$

It follows that $J[y] \geqq \operatorname{Min}\left(z^{\prime}-z^{2}-f\right)$ and this implies

$$
\lambda_{0} \geqq \operatorname{Min}\left(z^{\prime}-z^{2}-f\right) \text {. }
$$

Incidentally, this is an adaptation of Wintner's condition ([5], p. 368) to the eigenvalue problem under consideration. By different choices of $z$ we can obtain different lower bounds of $\lambda_{0}$.

First take as $z$ an indefinite integral $g$ of $f_{1} \equiv f-c_{0}$. Then $z$ is certainly periodic and (5) becomes

$$
\lambda_{0} \geqq \operatorname{Min}\left(-c_{0}-g^{2}\right)=-c_{0}-(\operatorname{Max}|g|)^{2} .
$$

To estimate $\operatorname{Max}|g|$, we introduce the oscillation $A$ of $g$ :

$$
A=\operatorname{Max} g-\operatorname{Min} g .
$$

Since $g$ is continuous, there are values $a, b$ of $x$ such that $g(a)=\operatorname{Min} g, g(b)=\operatorname{Max} g$. Since $g$ is periodic, we may assume $a<b<a+1$ without loss of generality. Then we have

Hence

$$
A=g(b)-g(a)=\int_{a}^{b} f_{1} d x=-\int_{b}^{a+1} f_{1} d x .
$$

$$
A \leqq \int_{a}^{b}\left|f_{1}\right| d x, \quad A \leqq \int_{b}^{a+1}\left|f_{1}\right| d x .
$$

Addition of both inequalities and application of the Schwarz inequality give

$$
2 A \leqq \int_{a}^{a+1}\left|f_{1}\right| d x=\int_{0}^{1}\left|f_{1}\right| d x \leqq\left[\int_{0}^{1} f_{1}^{2} d x\right]^{1 / 2} .
$$

Heretofore $g$ has been determined only up to an arbitrary additive constant. If we choose this constant appropriately, we can make $g(b)=-g(a)=A / 2$. Then we have Max $|g|=$ $A / 2$ and hence

$$
(\operatorname{Max}|g|)^{2} \leqq \frac{1}{16} \int_{0}^{1} f_{1}^{2} d x=\frac{1}{8} \sum_{n=1}^{\infty}\left|c_{n}\right|^{2}
$$

which, combined with (6), proves (3).

Another estimation of $\lambda_{0}$ is obtained by adjusting the arbitrary constant in $g$ in such a way that

$$
g(x)=\sum_{n \neq 0}(2 \pi i n)^{-1} c_{n} \exp (2 \pi i n x) .
$$


Then we have

and (6) gives

$$
\operatorname{Max}|g| \leqq \pi^{-1} \sum_{n=1}^{\infty} n^{-1}\left|c_{n}\right|
$$

$$
\lambda_{0} \geqq-c_{0}-\pi^{-2}\left(\sum_{n=1}^{\infty} n^{-1}\left|c_{n}\right|\right)^{2} .
$$

It will be noted that (3) and (7) are mutually independent. In any case, however, (7) is better than the second inequality of (2), for we have

$$
\pi^{-2}\left(\sum n^{-1}\left|c_{n}\right|\right)^{2} \leqq \pi^{-2} \sum n^{-2} \sum\left|c_{n}\right|^{2}=\frac{1}{6} \sum\left|c_{n}\right|^{2}
$$

by the Cauchy inequality. In the same way it is seen that (7) is better than (3) if $c_{1}=0$.

It is not clear whether the coefficient $1 / 8$ in (3) can still be replaced by a smaller one. However, it cannot be made smaller than $1 / 2 \pi^{2}$ (Cf. Putnam [3], p. 314, where the figure $1 / 4 \pi^{2}$ is given). In fact, consider the case $f(x)=2 c_{1} \cos 2 \pi x$ with a real $c_{1}$ (Mathieu equation); then the formula of the usual perturbation theory (Courant-Hilbert [1], p. 300) yields easily the expansion

$$
\lambda_{0}=-\left(2 \pi^{2}\right)^{-1} c_{1}^{2}+\cdots
$$

which is certainly convergent for sufficiently small value of $\left|c_{1}\right|$ (Kato [2], p. 169).

It will be noted that the lower bounds of $\lambda_{0}$ as given by the formulas (3) and (7) are, though rigorous, not very accurate from the practical standpoint. Especially this is the case when the Fourier coefficients $c_{n}$ are large, for we have

$$
\lambda_{0} \geqq-\operatorname{Max} f \geqq-c_{0}-2 \sum_{n=1}^{\infty}\left|c_{n}\right|,
$$

as is easily seen by setting $z=0$ in (5).

For more accurate estimation of $\lambda_{0}$ in individual cases, it is more convenient to use (5) directly. For instance consider the case $f=2 c_{1} \cos 2 \pi x$ stated above. If we set $z=k \sin 2 \pi x$, we have by (5)

$$
\begin{aligned}
\lambda_{0} & \geqq \operatorname{Min}\left[\left(2 \pi k-2 c_{1}\right) \cos 2 \pi x-k^{2} \sin ^{2} 2 \pi x\right] \\
& =\operatorname{Min}\left[\left(k \cos 2 \pi x+\pi-k^{-1} c_{1}\right)^{2}-k^{2}-\left(\pi-k^{-1} c_{1}\right)^{2}\right] \\
& \geqq-k^{2}-\left(\pi-k^{-1} c_{1}\right)^{2} .
\end{aligned}
$$

This is true for every $k$. If we assume $c_{1}>0$ and take $k=c_{1}^{1 / 2}$, we obtain

$$
\lambda_{0} \geqq-2 c_{1}+2 \pi c_{1}^{1 / 2}-\pi^{2} .
$$

It is easily seen that the right-hand side coincides with the asymptotic expansion of $\lambda_{0}$ for $c_{1} \rightarrow \infty$ up to the order $c_{1}^{1 / 2}$ inclusive (Strutt [4], p. 37, Eq. (4), where we have to set $\left.\lambda=\pi^{-2} \lambda_{0}, h^{2}=\pi^{-2} c_{1}, m_{1}=1\right)$.

\section{REFERENCES}

1. R. Courant and D. Hilbert, Methoden der Mathematischen Physik, I, second edition, Berlin, 1931 .

2. T. Kato, On the convergence of the perturbation method, J. Fac. Sci., Univ. Tokyo, (I) 6, 145-226 (1951).

3. C. R. Putnam, On the least eigenvalue of Hill's equation, Q. Appl. Math. 9, 310-314 (1951).

4. M. J. O. Strutt, Lamésche, Mathieusche und verwandte Funktionen in Physik und Technik, Berlin, 1932.

5. A. Wintner, On the non-existence of conjugate points, Amer. J. Math. 73, 368-380 (1951). 